\title{
RF Tomography for Below-ground Imaging of Extended Areas and Close-in Sensing
}

\author{
Lorenzo Lo Monte, Student Member, IEEE, Danilo Erricolo Senior Member, IEEE, \\ Francesco Soldovieri, Michael C. Wicks Fellow, IEEE
}

\begin{abstract}
Three extensions to RF Tomography for imaging of voids under extended areas of regard are presented.

These extensions are motivated by three challenges. One challenge is the lateral wave, which propagates in proximity of the air-earth interface, and represents the predominant radiation mechanism for wide area surveillance, sensing of denied terrains, or close-in sensing. A second challenge is the direct path coupling between $T x$ and $R x$, that affects the measurements. A third challenge is the generation of clutter by the unknown distribution of anomalies embedded in the ground.
\end{abstract}

These challenges are addressed and solved using the following strategies: 1) A forward model for RF Tomography that includes lateral waves expressed in closed-form (for fast computation); 2) a strategy that reduces the direct-path coupling between any $\mathbf{T x}$ Rx pair; 3) an improved inversion scheme that is robust with respect to noise, clutter, and high attenuation.

An FDTD simulation of a scenario representing close-in sensing of a denied area is performed, and reconstructed images obtained using the improved and the classical model of RF Tomography are compared.

Index Terms-Ground Penetrating Radar, RF Tomography, Tunnel Detection, Lateral Waves, Green's Functions.

\section{INTRODUCTION}

$\mathrm{T}$ HE problem of underground void detection is paramount to secure borders, sensitive areas, and for search \& rescue missions. To date, no underground imaging technique emerged as a standard for close-in sensing of wide denied areas, where minimal human intervention is required [1].

A promising strategy is introduced in [1], [7] where one set of transmitters (Tx) and one set of receivers ( $\mathrm{Rx}$ ) are placed on (or in) the ground at arbitrary positions. The transmitters (Tx) radiate a monochromatic signal, which impinges upon a buried

Manuscript Received on May $21^{\text {st }} 2009$, revised, published. This work was partially supported by the Air Force Research Laboratory, under contract \#F33601-02-F-A581.

Lorenzo Lo Monte is with General Dynamics Information Technology, 5100 Springfield Pike, Suite 504, Dayton, OH 45431, USA. Email: lorenzo.lomonte@gdit.com.

Danilo Erricolo is with the University of Illinois at Chicago, $851 \mathrm{~S}$. Morgan Ave, Chicago, IL 60607. Email : erricolo@ece.uic.edu.

Francesco Soldovieri is with the Istituto per il Rilevamento Elettromagnetico dell'Ambiente - CNR, 80124, Naples, Italy. Email: soldovieri.f@irea.cnr.it.

Michael C. Wicks is with the Air Force Research Laboratory, Sensors Directorate, 26 Electronic Parkway, Rome NY 13441. Email: michael.wicks@rl.af.mil.

Digital Object Identifier : dielectric or conductive anomaly, thus generating a scattered wave. Multiple receivers collect samples of the scattered electric field, and relay this information to a base station. Images of the below-ground scene are then reconstructed using the principles of $R F$ Tomography. Advantages and mathematical derivations of RF Tomography for underground imaging are discussed in [1]. The approach is technically valid for any sensor disposition and terrain shape, provided that the Green's function characterizing the problem is properly selected. In [1] the Green's function for a homogeneous space was applied, due to its simplicity of implementation. This choice has been proven to work satisfactorily when the sensors and targets are located nearly vertically above the targets, thus avoiding artefacts due to the discontinuity at the air-earth interface.

However, practical applications require wide areas of investigations (e.g. underground networks and facilities), denied areas (e.g. sensing of urban environment) or close-in sensing (e.g. covert missions). In these cases, sensors remotely probe underground regions at long ranges, and the propagation of waves occurs primarily along the air/ground interface; hence, the predominant propagation mode is the lateral wave [8]-[10]. Therefore, one contribution of this work is the introduction of a more accurate forward model by invoking a closed form Green's function that accounts for the air-earth discontinuity (see Appendix).

In addition, RF Tomography is based upon the knowledge of the scattered field from targets. In real cases, receivers are irradiated by a strong electromagnetic field due to the direct link between each $\mathrm{Tx}$ and $\mathrm{Rx}$ pair (i.e. direct path coupling [1]). Hence, as a second contribution, in Section III, an efficient technique that mitigates the direct path coupling (by joint $\mathrm{Tx}$ and $\mathrm{Rx}$ null steering) is presented.

Moreover, distributed anomalies (e.g. weathered soils) also generate a bias to the measured scattered field, which may be considered as clutter. A third contribution, given in Section IV, is an improvement upon the inversion schemes already discussed in literature [3]-[5], based on the findings described by Zhdanov [2] from the geophysical community; this improved method is more robust with respect to perturbations (e.g. clutter) of the measured scattered field.

The combination of these three new strategies improves the image reconstruction process, especially for large areas of interest, shallow targets, and close-in sensing, as shown in Section V. 


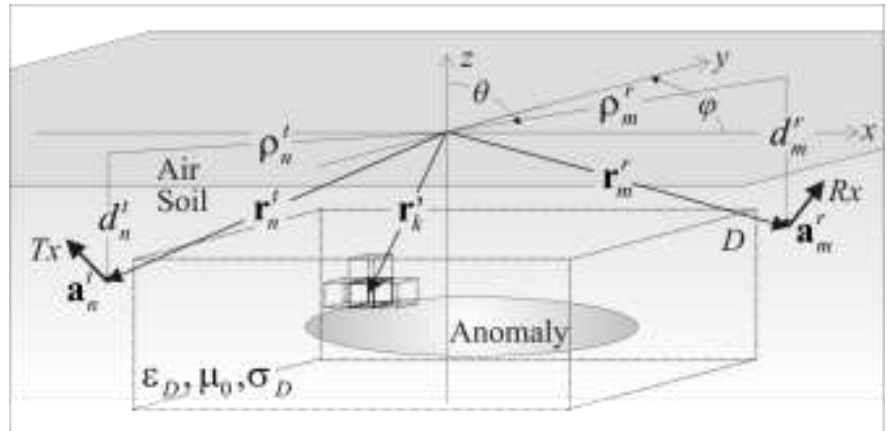

Figure 1: 3D Geometry for the model.

\section{FORWARD MODEL}

The 3D geometry depicted in Figure 1 is considered. Under the monochromatic assumption (single work frequency $f$ ), the air half-space is modeled as a free space medium, while the ground half-space is modeled as a homogeneous medium with relative dielectric permittivity $\varepsilon_{D}$, conductivity $\sigma_{D}$, and magnetic permeability $\mu_{0}$. The targets (i.e. tunnels, caches or voids) are assumed to reside in the investigation domain $D$. The sources are $N$ electrically small dipoles (of length $\Delta l$ ) fed with current $I$. For each transmitting sensor, the total field $\mathbf{E}$ is collected by $M$ receivers. Both transmitters and receivers reside inside the ground but outside the investigation domain $D$. The relative dielectric permittivity $\varepsilon_{r}\left(\mathbf{r}^{\prime}\right)$ and the conductivity $\sigma\left(\mathbf{r}^{\prime}\right)$ inside $D$ are the unknowns of this problem. The contrast function is defined as [1]

$$
\varepsilon_{\delta}\left(\mathbf{r}^{\prime}\right)=\varepsilon_{r}\left(\mathbf{r}^{\prime}\right)-\varepsilon_{D}+j\left(\sigma\left(\mathbf{r}^{\prime}\right)-\sigma_{D}\right) / 2 \pi f \varepsilon_{0} .
$$

However, other definitions may be used [3]-[5], [8]. By pointing out the first order Born approximation [2]-[5], [8] the field received by a dipole oriented along the direction $\mathbf{a}_{m}^{r}$, positioned at $\mathbf{r}_{m}^{r}$ due to a transmitting dipole oriented along the direction $\mathbf{a}_{n}^{t}$, positioned at $\mathbf{r}_{n}^{t}$, can be written as [1]:

$$
\begin{aligned}
& E\left(\mathbf{r}_{n}^{t}, \mathbf{r}_{m}^{r}\right)=Q \mathbf{a}_{m}^{r} \cdot \underline{\underline{\mathbf{G}}}\left(\mathbf{r}_{m}^{r}, \mathbf{r}_{n}^{t}\right) \cdot \mathbf{a}_{n}^{t}+H\left(\mathbf{r}_{n}^{t}, \mathbf{r}_{m}^{r}\right)+T+ \\
& Q k_{0}^{2} \iiint_{D}\left[\mathbf{a}_{m}^{r} \cdot \underline{\underline{G}}\left(\mathbf{r}_{m}^{r}, \mathbf{r}^{\prime}\right)\right] \cdot\left[\underline{\underline{G}}\left(\mathbf{r}^{\prime}, \mathbf{r}_{n}^{t}\right) \cdot \mathbf{a}_{n}^{t}\right] \varepsilon_{\delta}\left(\mathbf{r}^{\prime}\right) d \mathbf{r}^{\prime}
\end{aligned}
$$

where $Q=j \omega \mu_{0} \Delta l I$ for an electrically small dipole [3], the quantities $H$ (multiple scattering) and $T$ (random noise) represent unpredictable perturbations to the total field. $\mathbf{G}$ is the Green's dyad, to be chosen according to the adopted formulation. The first term in eq. (2), i.e.

$$
Q \mathbf{a}_{m}^{r} \cdot \underline{\underline{G}}\left(\mathbf{r}_{m}^{r}, \mathbf{r}_{n}^{t}\right) \cdot \mathbf{a}_{n}^{t}
$$

describes the direct path coupling between a particular Tx and Rx pair. The cancellation of this coupling from the measured field is a critical problem in RF Tomography: although it can be analytically predicted (and cancelled) using (3), in practical cases its magnitude may be up to $50-60 \mathrm{~dB}$ higher than the scattered signal. In these conditions, the dynamic range of the receiver's amplifiers may not be large enough, or the quantization steps may not be as fine as required to sample both scattered and direct-path coupling fields accordingly. Clearly, the best solution is to cancel the direct-path contribution before it reaches the receiver.

\section{Direct Path Coupling Mitigation}

In this section, a direct-path coupling mitigation technique is introduced. The key factor is the possibility to steer the Tx and Rx dipoles toward desired directions. Rotation of dipoles may be performed using mechanical devices, or by properly feeding a set of co-located orthogonal dipoles (see [1]). The proposed strategy properly steers the Tx dipole in order to minimize the field at receiver side, and then turns the Rx dipole in order to be orthogonal to the expected direct path electric field. Mathematically, these rotations are computed by solving a series of constrained minimization problems, for each $\mathrm{Tx}$ and Rx pair:

minimize $\left\|\underline{\underline{G}}\left(\mathbf{r}_{m}^{r}, \mathbf{r}_{n}^{t}\right) \cdot \mathbf{a}_{n}^{t}\right\|_{2}^{2}$ subject to $\left(\mathbf{a}_{n}^{t}\right)^{T} \cdot \mathbf{a}_{n}^{t}=1$ (4)

In Lagrangian form it becomes:

$$
\begin{aligned}
\Lambda_{n, m}\left(\mathbf{a}_{n}^{t}, \lambda\right)= & \left(\mathbf{a}_{n}^{t}\right)^{T} \cdot \underline{\underline{G}}^{H}\left(\mathbf{r}_{m}^{r}, \mathbf{r}_{n}^{t}\right) \cdot \underline{\underline{\mathbf{G}}}\left(\mathbf{r}_{m}^{r}, \mathbf{r}_{n}^{t}\right) \cdot \mathbf{a}_{n}^{t} \\
& -\lambda\left[\left(\mathbf{a}_{n}^{t}\right)^{T} \cdot \mathbf{a}_{n}^{t}-1\right]
\end{aligned}
$$

where $\mathbf{G}^{H}$ denotes the Hermitian of $\mathbf{G}$. By imposing $\nabla \Lambda_{n, m}=0$ we obtain:

$$
\underline{\underline{\mathbf{G}}}^{H}\left(\mathbf{r}_{m}^{r}, \mathbf{r}_{n}^{t}\right) \cdot \underline{\underline{\mathbf{G}}}\left(\mathbf{r}_{m}^{r}, \mathbf{r}_{n}^{t}\right) \cdot \mathbf{a}_{n}^{t}=\lambda \mathbf{a}_{n}^{t}
$$

Therefore, the $\mathbf{a}_{n}^{t}$ direction that minimizes the power at a desired location is the eigenvector associated with the smallest eigenvalue of the matrix $\mathbf{G}^{H} \mathbf{G}$. Similarly, this minimization can be applied at the receiver side. Defining the vector

$$
\underline{E}_{\min }=\underline{\underline{\mathbf{G}}}\left(\mathbf{r}_{m}^{r}, \mathbf{r}_{n}^{t}\right) \cdot \mathbf{a}_{n}^{t}
$$

as the electric field obtained when $\mathbf{a}_{n}^{t}$ is chosen according to (4), a second minimization problem can be formulated as 
minimize $\left\|\left(\mathbf{a}_{m}^{r}\right)^{T} \cdot \underline{E}_{\min }\right\|_{2}^{2}$ subject to $\left(\mathbf{a}_{m}^{r}\right)^{T} \cdot \mathbf{a}_{m}^{r}=1$.

The minimization is achieved when $\mathbf{a}_{m}^{r}$ is chosen to be the eigenvector corresponding to the smallest eigenvalue of the matrix $E_{\min } \cdot\left(E_{\min }\right)^{T}$. If the dipole can be steered only over a horizontal plane (e.g. by using two crossed dipoles), the steering directions are easily obtained by setting to zero the $z$ component of each vector $\mathbf{a}$.

As tested via numerical analysis, the application of these strategies guarantees an acceptable minimization of the received signal due to direct-path coupling. Therefore, the total electric field can be reasonably approximated only with the scattered contribution from targets inside the region $D$, i.e. [3]:

$$
\begin{aligned}
& E\left(\mathbf{r}_{n}^{t}, \mathbf{r}_{m}^{r}\right) \cong E^{S}\left(\mathbf{r}_{n}^{t}, \mathbf{r}_{m}^{r}\right)=\mathbf{L}\left(\varepsilon_{\delta}\left(\mathbf{r}^{\prime}\right)\right)=Q k_{0}^{2} \times \\
& \iiint_{D}\left[\mathbf{a}_{m}^{r} \cdot \underline{\underline{G}}\left(\mathbf{r}_{m}^{r}, \mathbf{r}^{\prime}\right)\right] \cdot\left[\underline{\underline{G}}\left(\mathbf{r}^{\prime}, \mathbf{r}_{n}^{t}\right) \cdot \mathbf{a}_{n}^{t}\right] \varepsilon_{\delta}\left(\mathbf{r}^{\prime}\right) d \mathbf{r}^{\prime}
\end{aligned}
$$

\section{INVERSION}

Assuming that the clutter contribution has been completely mitigated, and the field received by the sensors is given by the forward model in eq. (9), the sampled field at each Tx and Rx pair can be collected in a vector $\underline{E}=\left\{E\left(\mathbf{r}_{n}^{t}, \mathbf{r}_{m}^{r}\right)\right\}$, and the investigation region $D$ can be discretized in $K$ voxels, each one located at position $\mathbf{r}_{k}{ }^{\prime}$ : the contrast function can be represented in a column vector $\underline{\varepsilon}_{\delta}=\left\{\varepsilon_{\delta}\left(\mathbf{r}_{k}^{\prime}\right)\right\}$. After this discretization, eq. (9) can be reformulated in matrix form:

$$
\underline{E}=\underline{\underline{\mathbf{L}}} \underline{\varepsilon}_{\delta}
$$

where $\mathbf{L}$ is generally a ill-conditioned matrix.

Several methods have been proposed to solve (10), such as back-propagation [1], truncated singular value decomposition [4], [11], and Tikhonov regularization [2], [11]. In this work, we introduce a refined version of Tikhonov regularization that equalizes the sensitivity of each Tx and Rx pair by selecting a proper weighting factor, and introduces a term able to exploit the a priori information on the values of the dielectric anomalies. Accordingly, the contrast function (as a function of the regularization parameter $\beta$ ) can be estimated [2]:

$$
\underline{\hat{\varepsilon}}_{\delta}(\beta)=\left(\mathbf{L}^{H} \mathbf{W}_{E}^{2} \mathbf{L}+\beta \mathbf{W}_{\varepsilon}^{2}\right)^{-1}\left(\mathbf{L}^{H} \mathbf{W}_{E}^{2} \underline{E}+\beta \mathbf{W}_{\varepsilon}^{2} \underline{\varepsilon}_{\delta}^{0}\right)
$$

where $\underline{\varepsilon}_{\delta}^{0} \quad$ represents the known dielectric anomalies embedded in region $D$, and

$$
\mathbf{W}_{\varepsilon}=\operatorname{diag}\left(\mathbf{L}^{*} \mathbf{L}\right)^{1 / 2}, \mathbf{W}_{E}=\operatorname{diag}\left(\mathbf{L} \mathbf{L}^{*}\right)^{1 / 2}
$$

are (diagonal) weighting matrices opportunely defined in order to minimize the sensitivity of the system [2].

In most cases, the weighting matrices have a small dynamic range. Therefore, we can approximate $\mathbf{W}_{\varepsilon} \cong \alpha \mathbf{I}$ in eq. (11). If we perform the singular value decomposition [11] of a properly defined weighed matrix $\underline{\underline{\mathbf{L}}}_{w}=\mathbf{W}_{E} \mathbf{L}=\underline{\underline{\mathbf{U}}} \underline{\underline{\mathbf{S}}} \underline{\underline{\mathbf{V}}}^{H}$, eq. (11) becomes

$$
\begin{aligned}
\underline{\hat{\varepsilon}}_{\delta} & =\left(\mathbf{L}_{w}^{*} \mathbf{L}_{w}+\beta \mathbf{W}_{\varepsilon}^{2}\right)^{-1}\left(\mathbf{L}_{w}^{*} \mathbf{W}_{E} \underline{E}+\beta \mathbf{W}_{\varepsilon}^{2} \underline{\varepsilon}_{\delta}^{0}\right) \\
& \cong\left(\mathbf{V S}^{*} \mathbf{S} \mathbf{V}^{*}+\alpha^{2} \beta \mathbf{I}\right)^{-1}\left(\mathbf{V} \mathbf{S}^{*} \mathbf{U}^{*} \mathbf{W}_{E} \underline{E}+\beta \mathbf{W}_{\varepsilon}^{2} \underline{\varepsilon}_{\delta}^{0}\right) \\
& =\mathbf{V} \operatorname{diag}\left(s_{i}^{2}+\alpha^{2} \beta\right)^{-1}\left(\mathbf{S}^{*} \mathbf{U}^{*} \mathbf{W}_{E} \underline{E}+\mathbf{V}^{*} \beta \mathbf{W}_{\varepsilon}^{2} \underline{\varepsilon}_{\delta}^{0}\right)
\end{aligned}
$$

where $S_{i}$ represent the $i$-th singular value of $\mathbf{L}_{w}$. The advantage obtained in applying eq. (13) is that the contrast function as function of $\beta$ can be computed via (fast) matrix multiplications.

\section{Simulations AND CONCLUSIONS}

A simulation is presented in order to demonstrate the improvements achieved by using 1) lateral waves in the forward model, 2) direct-path mitigation, 3) weighted Tikhonov regularization. The test scene represents a situation where sensors are surrounding the wide (denied) area of interest (close-in sensing), and probe the region $D$ mostly via lateral waves (see Fig. 2 for details).

The targets are two hollow cylinders emulating two tunnels, located with their axes parallel to the surface and at a depth $d^{\prime}=5 \mathrm{~m}$ below the ground (with respect to their center) having $\varepsilon_{D}=9$, and $\sigma_{D}=5 \times 10^{-4} \mathrm{~S} / \mathrm{m}$. No a priori information about dielectric anomalies in the scene is considered, thus $\underline{\varepsilon}_{\delta}^{0}=0$. The work frequency is $5 \mathrm{MHz} .12$ transmitters and 20 receivers are placed along a circle encompassing the two tunnels, as illustrated in Fig. 2. Each sensor is emplaced at depth $d=0.25 \mathrm{~m}$ beneath the surface. The received electric field has been synthesized using the FDTD simulator GPRMAX [6] for each Tx and Rx pair.

In the first simulation, 1) the homogeneous Green's function [8-9] having the properties of the soil is inserted in the forward model; 2) the Truncated SVD method [1], [4] is used for the inversion; and, 3) the direct-path coupling assumed completely cancelled. The reconstruction result is shown in Fig. 3.

In the second simulation, 1) the closed-form Green's function that accounts for the lateral wave (see Appendix) was used; 2) the weighted Tikhonov method proposed in Section IV was implemented; and, 3) the direct-path mitigation algorithm (Section III) was used to choose the direction of each sensor. The reconstruction result is shown in Fig. 4. 


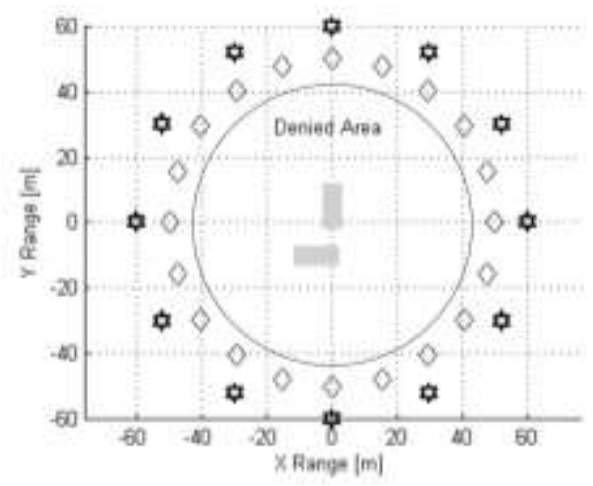

Figure 2: Geometry for the simulation: transmitters and receivers are indicated with stars and diamonds respectively, and the two tunnels are located at the center of the scene.

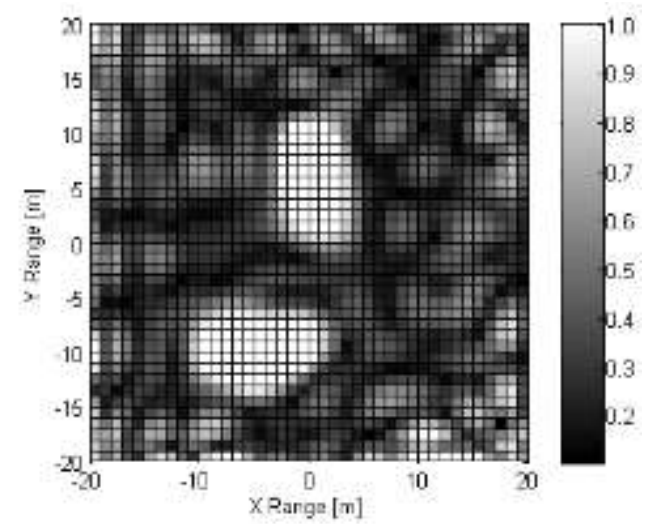

Figure 3: Reconstructed image using homogeneous Green's function. Depth slice: $5 \mathrm{~m}$.

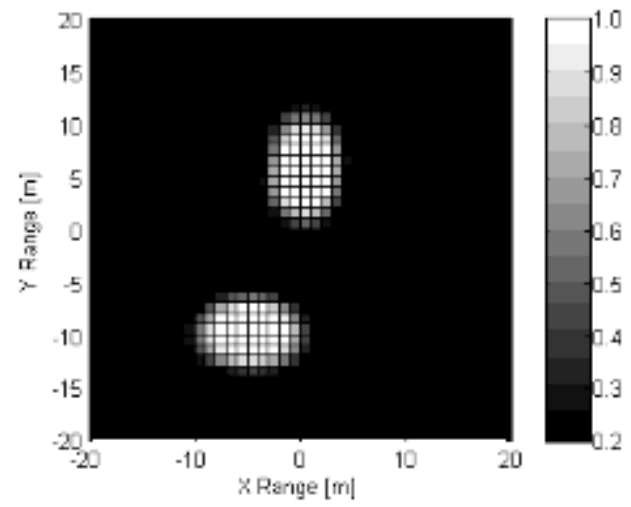

Figure 4: Reconstructed image using half-space Green's function. Depth slice: $5 \mathrm{~m}$.

As expected, the shallow targets are mainly irradiated by the lateral wave excited at the air-earth interface, and the classical Green's function for the homogeneous space cannot accurately predict the field value. Conversely, the half-space Green's function generates highly resolved images, especially for shallow targets, since it accounts for the effects of lateral wave (see Fig. 4). Therefore, we conclude that when RF tomography is applied in wide areas, denied terrains, or when targets are in shallow regions, the discussed improvements (i.e. the inclusion of the lateral waves propagation within the realm of $\mathrm{RF}$ tomography and its fast numerical computation, the direct-path coupling suppression using steerable radiators, the improved inversion scheme for better handling the clutter) are a suitable choice to obtain high quality reconstructions.

\section{APPENDIX: HALF SPACE GREEN'S FUNCTION}

Half-space Green's functions have been proposed in the literature [3], [5], [9], [11], but they are generally expressed as asymptotic expansions, or in spectral form. Nevertheless, King [10] derived explicit closed form expressions for the electric field generated by horizontal and vertical dipoles buried in a lossy medium (under assumption reported in (15)). From King's formulas, a closed form expression for the half space Green's function is derived (valid only when antennas and targets are embedded in the soil).

A dyadic Green's function can be expressed as follows:

$$
\underline{\underline{\mathbf{G}}}\left(\mathbf{r}, \mathbf{r}^{\prime}\right)=-\frac{j}{4 \pi k_{D}^{2}}\left[\begin{array}{lll}
g_{X X} & g_{X Y} & g_{X Z} \\
g_{Y X} & g_{Y Y} & g_{Y Z} \\
g_{Z X} & g_{Z Y} & g_{Z Z}
\end{array}\right]
$$

In this case, $\mathbf{r}$ represents the observation point, and $\mathbf{r}$ 'represent the current (physical or equivalent) source position. The coefficients in (14) are given:

$$
\begin{array}{ll}
g_{X X}=g_{\rho}^{h} \cos ^{2} \varphi-g_{\varphi}^{h} \sin ^{2} \varphi & g_{X Y}=\left(g_{\rho}^{h}+g_{\varphi}^{h}\right) \sin \varphi \cos \varphi \\
g_{X Z}=-g_{\rho}^{v} \cos \varphi & g_{Y X}=\left(g_{\rho}^{h}+g_{\varphi}^{h}\right) \cos \varphi \sin \varphi \\
g_{Y Y}=g_{\rho}^{h} \sin ^{2} \varphi-g_{\varphi}^{h} \cos ^{2} \varphi & g_{Y Z}=-g_{\rho}^{v} \sin \varphi \\
g_{Z X}=-g_{z}^{h} \cos \varphi & g_{Z Y}=-g_{z}^{h} \sin \varphi \quad g_{Z Z}=g_{z}^{v}
\end{array}
$$

Using the following symbols (see Fig. 1) and conditions:

$$
\begin{gathered}
k_{0}=\omega \sqrt{\mu_{0} \varepsilon_{0}} \quad k_{D}=\omega \sqrt{\mu_{0} \varepsilon_{0} \varepsilon_{D}} \\
d=-z \quad d^{\prime}=-z^{\prime} \quad \rho=\sqrt{\left(x-x^{\prime}\right)^{2}+\left(y-y^{\prime}\right)^{2}} \\
r_{d}=\left|\mathbf{r}-\mathbf{r}^{\prime}\right|=\sqrt{\rho^{2}+\left(d-d^{\prime}\right)^{2}} \quad r_{i}=\sqrt{\rho^{2}+\left(d+d^{\prime}\right)^{2}} \\
\left|k_{D}\right| \geq 3 k_{0} \quad\left|k_{D} \rho\right|>3
\end{gathered}
$$

the Green's function coefficients are expressed as follows:

$$
\begin{aligned}
& g_{\rho}^{h}=e^{j k_{D} r_{d}}\left[\frac{2 k_{D}}{r_{d}^{2}}+\frac{2 j}{r_{d}^{3}}+\frac{\left(d-d^{\prime}\right)^{2}}{r_{d}^{2}}\left(\frac{j k_{D}^{2}}{r_{d}}-\frac{3 k_{D}}{r_{d}^{2}}-\frac{3 j}{r_{d}^{3}}\right)\right]+ \\
& e^{j k_{D_{i}} r_{i}}\left[\frac{2 k_{D}}{r_{i}^{2}}+\frac{2 j}{r_{i}^{3}}+\frac{\left(d+d^{\prime}\right)^{2}}{r_{i}^{2}}\left(\frac{j k_{D}^{2}}{r_{i}}-\frac{3 k_{D}}{r_{i}^{2}}-\frac{3 j}{r_{i}^{3}}\right)\right] \\
& -2 e^{j k_{D} r_{i}}\left[\frac{k_{D}}{r_{i}^{2}}+\frac{j}{r_{i}^{3}}+\left(\frac{d+d^{\prime}}{\rho}\right)\left(\frac{j k_{D}}{\rho^{2}}-\frac{3}{2 \rho^{3}}\right)\right]-2 k_{0} \times \\
& {\left[\frac{j k_{0}}{\rho}-\frac{1}{\rho^{2}}-\frac{j}{k_{0} \rho^{3}}-\frac{k_{0}^{3}}{k_{D}}\left(\frac{\pi}{k_{0} \rho}\right)^{1 / 2} e^{-j p} F(p)\right] e^{j k_{D}\left(d+d^{\prime}\right)} e^{j k_{0} \rho}}
\end{aligned}
$$




$$
\begin{aligned}
& g_{\varphi}^{h}=-e^{j k_{D} r_{d}}\left(\frac{j k_{D}^{2}}{r_{d}}-\frac{k_{D}}{r_{d}^{2}}-\frac{i}{r_{d}^{3}}\right)-e^{j k_{D} r_{i}}\left(\frac{j k_{D}^{2}}{r_{i}}-\frac{k_{D}}{r_{i}^{2}}-\frac{j}{r_{i}^{3}}\right) \\
& +4 e^{j k_{D} r_{i}}\left(\frac{j k_{D}^{2}}{2 r_{i}}-\frac{k_{D}}{r_{i}^{2}}-\frac{j}{r_{i}^{3}}\right)+2 j e^{j k_{D} r_{i}}\left(\frac{d+d^{\prime}}{r_{i}}\right) \times \\
& \left(\frac{j k_{D}^{2}}{\rho}-\frac{3 k_{D}}{2 \rho^{2}}-\frac{5 j}{8 \rho^{3}}\right)-2 e^{j k_{r_{i}}}\left(\frac{d+d^{\prime}}{r_{i}}\right)^{2} \times \\
& \left(\frac{j k_{D}^{2}}{r_{i}}-\frac{3 k_{D}}{r_{i}^{2}}-\frac{3 j}{r_{i}^{3}}\right)+4 e^{j k_{D}\left(d+d^{\prime}\right)} e^{j k_{0} \rho} \times \\
& {\left[\frac{k_{0}}{\rho^{2}}+\frac{j}{\rho^{3}}+\frac{j k_{0}^{3}}{2 k_{D} \rho}\left(\frac{\pi}{k_{0} \rho}\right)^{1 / 2} e^{-j p} F(p)\right]} \\
& g_{z}^{h}=-e^{j k_{D} r_{d}}\left(\frac{\rho}{r_{d}}\right)\left(\frac{d-d^{\prime}}{r_{d}}\right)\left(\frac{j k_{D}^{2}}{r_{d}}-\frac{3 k_{D}}{r_{d}^{2}}-\frac{3 j}{r_{d}^{3}}\right) \\
& -e^{j k_{D} r_{i}}\left(\frac{\rho}{r_{i}}\right)\left(\frac{d+d^{\prime}}{r_{i}}\right)\left(\frac{j k_{D}^{2}}{r_{i}}-\frac{3 k_{D}}{r_{i}^{2}}-\frac{3 j}{r_{i}^{3}}\right) \\
& +\frac{2 j k_{0}^{2}}{k_{D}} e^{j k_{D} r_{i}}\left(\frac{1}{\rho^{2}}-\frac{3 j}{2 k_{D} \rho^{3}}\right)-\frac{2 k_{0}^{2}}{k_{D}} e^{j k_{D}\left(d+d^{\prime}\right)} e^{j k_{0} \rho} \times \\
& \left(\frac{j k_{0}}{\rho}-\frac{1}{\rho^{2}}-\frac{k_{0}^{3}}{k_{D}}\left(\frac{\pi}{k_{0} \rho}\right)^{1 / 2} e^{-i p} F(p)\right) \\
& g_{\rho}^{v}=-e^{j k_{p_{d}} r_{d}}\left(\frac{j k_{D}^{2}}{r_{d}}-\frac{3 k_{D}}{r_{d}^{2}}-\frac{3 j}{r_{d}^{3}}\right)\left(\frac{\rho}{r_{d}}\right)\left(\frac{d-d^{\prime}}{r_{d}}\right) \\
& +e^{j k_{D} r_{i}}\left(\frac{j k_{D}^{2}}{r_{i}}-\frac{3 k_{D}}{r_{i}^{2}}-\frac{3 j}{r_{i}^{3}}\right)\left(\frac{\rho}{r_{i}}\right)\left(\frac{d+d^{\prime}}{r_{i}}\right) \\
& +\frac{2 j k_{0}^{2}}{k_{D}} e^{j k_{D} r_{i}}\left(\frac{1}{\rho^{2}}+\frac{3 j}{2 k_{D} \rho^{3}}\right)-\frac{2 k_{0}^{2}}{k_{D}} e^{j k_{D}\left(d+d^{\prime}\right)} e^{j k_{0} \rho} \times \\
& \left(\frac{j k_{0}}{\rho}-\frac{1}{\rho^{2}}-\frac{k_{0}^{3}}{k_{D}}\left(\frac{\pi}{k_{0} \rho}\right)^{1 / 2} e^{-i p} F(p)\right) \\
& g_{z}^{v}=\left[\frac{j k_{D}^{2}}{r_{d}}-\frac{k_{D}}{r_{d}^{2}}-\frac{j}{r_{d}^{3}}-\left(\frac{d-d^{\prime}}{r_{d}}\right)^{2}\left(\frac{j k_{D}^{2}}{r_{d}}-\frac{3 k_{D}}{r_{d}^{2}}-\frac{3 j}{r_{d}^{3}}\right)\right] \times \\
& e^{j k_{D} r_{d}}-2 \frac{j k_{0}^{2}}{k_{D}^{2}} e^{j k_{k_{i}} r_{i}}\left(\frac{d+d^{\prime}}{\rho}\right)\left(\frac{j k_{D}^{2}}{\rho}-\frac{k_{D}}{2 \rho^{2}}+\frac{7 j}{8 \rho^{3}}\right) \\
& -e^{j k_{D} r_{i}}\left[\frac{j k_{D}^{2}}{r_{i}}-\frac{k_{D}}{r_{i}^{2}}-\frac{j}{r_{i}^{3}}-\left(\frac{d+d^{\prime}}{r_{i}}\right)^{2}\left(\frac{j k_{D}^{2}}{r_{i}}-\frac{3 k_{D}}{r_{i}^{2}}-\frac{3 j}{r_{i}^{3}}\right)\right]+ \\
& 2 \frac{k_{0}^{3}}{k_{D}^{2}} e^{j k_{D}\left(d+d^{\prime}\right)} e^{j k_{0} \rho}\left(\frac{j k_{0}}{\rho}-\frac{1}{\rho^{2}}-\frac{j k_{0}}{\rho^{3}}-\frac{k_{0}^{3}}{k_{D}}\left(\frac{\pi}{k_{0} \rho}\right) e^{-i p} F(p)\right)
\end{aligned}
$$

$$
F(p)=\frac{1}{2}(1+j)-\int_{0}^{p} \frac{1}{\sqrt{2 \pi t}} e^{j t} d t \quad p=\frac{k_{0}^{3} \rho}{2 k_{D}^{2}}
$$

Although eq. (16) is expressed in integral form, its range is limited by the maximum and minimum values of $\rho$ so that (16) can be easily approximated via a polynomial interpolation, or tabulated.

The advantage of using this formulation rather than the spectral representations [8-9] is that the Green's function computation time for each $\left(\mathbf{r}, \mathbf{r}^{\prime}\right)$ pair is comparable with the case of free space, since the expressions, although lengthy, are still in closed form.

\section{ACKNOWLEDGMENT}

The authors are thankful to Mr. W. J. Baldygo, Air Force Research Laboratory, and Dr. J. A. Sjogren, Air Force Office of Scientific Research, for sponsoring this research (AFRL Contract \#F33601-02-F-A58, DoD Grant \#FA9550-05-10443.

\section{REFERENCES}

[1] L. Lo Monte, D. Erricolo, F. Soldovieri, M. C. Wicks, "Radio Frequency Tomography for Tunnel Detection", IEEE Trans. Geosci. Remote Sens., in press.

[2] M. S. Zhdanov, Geophysical Inverse Theory and Regularization Problems, Methods in Geochemistry and Geophysics, Vol. 36, Elsevier, Amsterdam, 2002.

[3] T. J. Cui, and W. C. Chew, "Diffraction Tomographic Algorithm for the Detection of Three-Dimensional Objects Buried in a Lossy Half-Space," IEEE Trans. Antennas Propag., Vol. 50, No. 1, pp. 42-49, Jan. 2002.

[4] G. Leone, and F. Soldovieri, "Analysis of the Distorted Born Approximation for Subsurface Reconstruction: Truncation and Uncertainities Effect," IEEE Trans. Geosci. Remote Sens., Vol. 41, No. 1, pp. 66-74, Jan. 2003.

[5] P. Meincke, "Linear GPR Inversion for Lossy Soil and a Planar Air-Soil Interface," IEEE Trans. Geosci. Remote Sens., Vol. 39, No. 12, pp. 2713-2721, Dec. 2001.

[6] A. Giannopoulos, GPRMAX Simulator, www.gprmax.org

[7] J. Norgard, M. C. Wicks, and A. Drozd, "Distributed/Embedded SubSurface Sensors for Imaging Buried Objects with Reduced Mutual Coupling and Suppressed Electromagnetic Emissions," Proc. International Conference on Electromagnetics in Advanced Applications (ICEAA), pp. 427-430, Turin, Sept. 17-21, 2007.

[8] W. C. Chew, Waves and Fields in Inhomogeneous Media, IEEE Press, Piscataway NJ, 1995.

[9] C. T. Tai, Dyadic Green Functions in Electromagnetic Theory, Second Edition, IEEE Press, Piscataway, NJ, 1993.

[10] R. W. P. King, M. Owens, and T. T. Wu, Lateral Electromagnetic Waves. Springer-Verlag, New York, NY, 1992.

[11] P. C. Hansen, Rank Deficient and Discrete Ill-Posed Problems, SIAM, Philadelphia, PA, 1998. 